\title{
Analytical Model of a PZT Thick-Film Triaxial Accelerometer for Optimum Design
}

Hindrichsen, Christian Carstensen; Almind, Ninia Sejersen; Brodersen, S. H.; Hansen, Ole; Thomsen, Erik Vilain

Published in:

IEEE Sensors Journal

Link to article, DOI:

10.1109/JSEN.2009.2014412

Publication date:

2009

Document Version

Publisher's PDF, also known as Version of record

Link back to DTU Orbit

Citation (APA):

Hindrichsen, C. C., Almind, N. S., Brodersen, S. H., Hansen, O., \& Thomsen, E. V. (2009). Analytical Model of a PZT Thick-Film Triaxial Accelerometer for Optimum Design. IEEE Sensors Journal, 9(4), 419-429.

https://doi.org/10.1109/JSEN.2009.2014412

\section{General rights}

Copyright and moral rights for the publications made accessible in the public portal are retained by the authors and/or other copyright owners and it is a condition of accessing publications that users recognise and abide by the legal requirements associated with these rights.

- Users may download and print one copy of any publication from the public portal for the purpose of private study or research.

- You may not further distribute the material or use it for any profit-making activity or commercial gain

- You may freely distribute the URL identifying the publication in the public portal

If you believe that this document breaches copyright please contact us providing details, and we will remove access to the work immediately and investigate your claim. 


\title{
Analytical Model of a PZT Thick-Film Triaxial Accelerometer for Optimum Design
}

\author{
Christian Carstensen Hindrichsen, Ninia S. Almind, Simon H. Brodersen, Ole Hansen, and Erik Villain Thomsen
}

\begin{abstract}
We present a mechanical model of a triaxial micro accelerometer design using PZT thick-film as the sensing material. The model is based on the full anisotropic material tensors and Eulers' beam equation using simplifying assumptions where the smaller stress contributions are ignored. The model results in design equations that are useful for optimization of the sensitivity and bandwidth and for matching the sensitivity in the three orthogonal directions. A finite-element method (FEM) model is used to verify the analytical model. Equal sensitivity in all three geometrical directions is preferred, which induces a number of limitations in design parameter space, this is used in a design strategy for setting the six independent dimensions of the accelerometer. Design methods for optimum sensitivity of each axis and for equal sensitivity between different axes have been proposed.
\end{abstract}

Index Terms-Accelerometer, analytical model, PZT, thick-film, triaxial.

\section{INTRODUCTION}

$\mathbf{M}$ ICROELECTROMECHANICAL SYSTEM (MEMS) accelerometers based on the piezoelectric effect have received increasing attention throughout the last years [1]. A need for more cost efficient and miniaturized accelerometers increases the interest for MEMS-based solutions [2]. The piezoelectric accelerometer may show less temperature dependence, better long term stability and higher bandwidth compared to the two other main read out principles: capacitive and piezoresistive [3] depending, however, strongly on the properties of the piezoelectric material. Moreover, piezoelectric devices are self-generating and thus do not need a stable drive voltage to be applied. Applications for piezoelectric accelerometers would, therefore, typically be for machine monitoring or other application requiring high bandwidth [4].

Uniaxial accelerometers have worse cross axis sensitivity than a triaxial accelerometer with a single seismic mass, which therefore is preferred compared to three single uniaxial accelerometers when monitoring a 3-D acceleration. A triaxial

Manuscript received July 02, 2008; revised August 21, 2008; accepted August 26, 2008. Current version published February 27, 2009. This research is a collaboration between The Department of Micro and Nanotechnology (DTU Nanotech) and the two companies InSensor A/S and Ferroperm Piezoceramics A/S. This work is part of the PiMEMS Project which is supported in part by The Danish National Advanced Technology Foundation. Center for Individual Nanoparticle Functionality (CINF) is sponsored by The Danish National Research Foundation. The associate editor coordinating the review of this paper and approving it for publication was Prof. Gerald Gerlach.

The authors are with the Department of Micro- and Nanotechnology, Technical University of Denmark, DTU Nanotech, DK-2800 Kongens Lyngby, Denmark (e-mail: christian.hindrichsen@nanotech.dtu.dk; s042326@student.dtu.dk; s042275@student.dtu.dk; ole.hansen@nanotech.dtu.dk; erik.v. thomsen@nanotech.dtu.dk).

Digital Object Identifier 10.1109/JSEN.2009.2014412

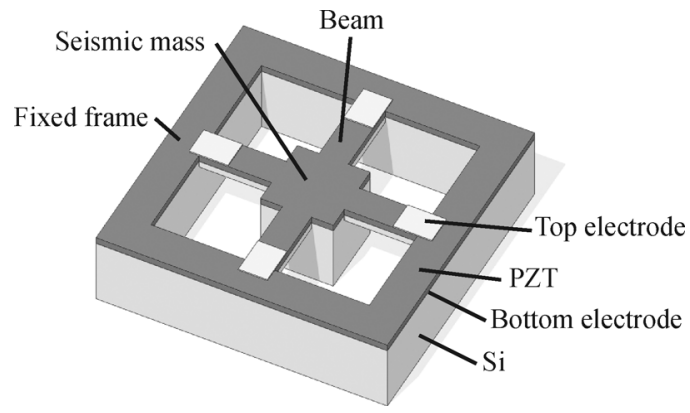

Fig. 1. The figure shows a conceptual drawing of the triaxial accelerometer. A central seismic mass is suspended by four beams. PZT is sandwiched between a bottom electrode covering the whole surface and a top electrode on each beam.

accelerometer has a more complex design and requires comparable sensitivity for the three sensing directions in order to be able to distinguish the three components of the 3 -D acceleration vector.

Single mass triaxial accelerometers have been reported in [5], but these do not have equal sensitivity for the three directions. An analytical model is a strong tool to find an optimal design resulting in equal sensitivity for all three directions. Both dynamic and static mechanical models have been presented in [6]-[8] but all describing accelerometers utilizing thin film and not PZT thick-film. These models all neglect the mechanical influence of the PZT. However, this assumption is not valid for thick-film accelerometers where the thickness of the PZT and the supporting silicon beam are comparable in size.

Accelerometers based on PZT thick-film have a higher voltage sensitivity for a given total beam thickness compared to thin-film devices since the generated voltage increases with the PZT thickness. An accelerometer based on PZT thick-film has been presented in [9], [10] but so far no mechanical model of the triaxial behavior of a thick-film-based accelerometer has been presented.

\section{ACCELEROMETER DESIGN}

Fig. 1 shows the accelerometer design with PZT on the bottom electrode covering the whole surface in the Si/PZT interface and four individual top electrodes. The design considered consists of a central seismic mass suspended by four beams $\left(b_{1}, b_{2}, b_{3}, b_{4}\right)$, as shown in Fig. 2. The design is based on screen printing of PZT thick-film on a patterned $\mathrm{Si}$ substrate. The screen printing technique allows for PZT thicknesses ranging from $\sim 15$ to $100 \mu \mathrm{m}$. The piezoelectric PZT is polarized orthogonal to the Si wafer plane. The important dimensions of the accelerometer are the length, width, and 


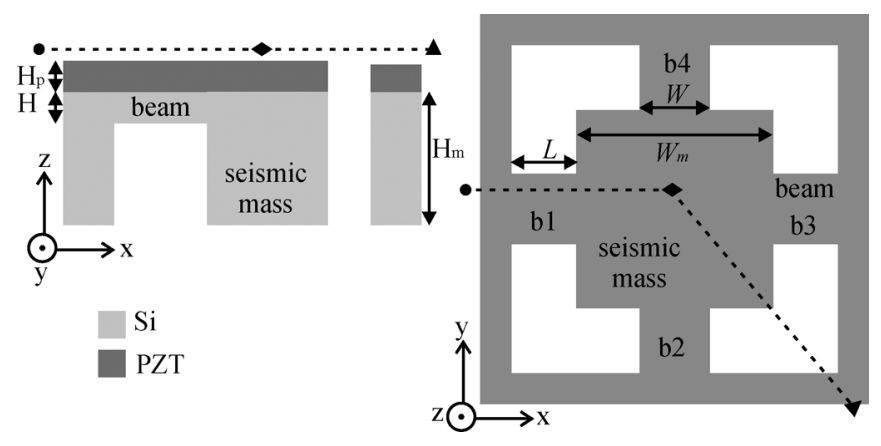

Fig. 2. Cross-sectional view (left) and top view (right) of the accelerometer including the six important dimensions is seen. The accelerometer consists of a seismic mass suspended by four beams named $\mathrm{b}_{1}-\mathrm{b}_{4}$.

TABLE I

ACCELEROMETER DESIGN PARAMETERS AND THEIR ABBREVIATIONS

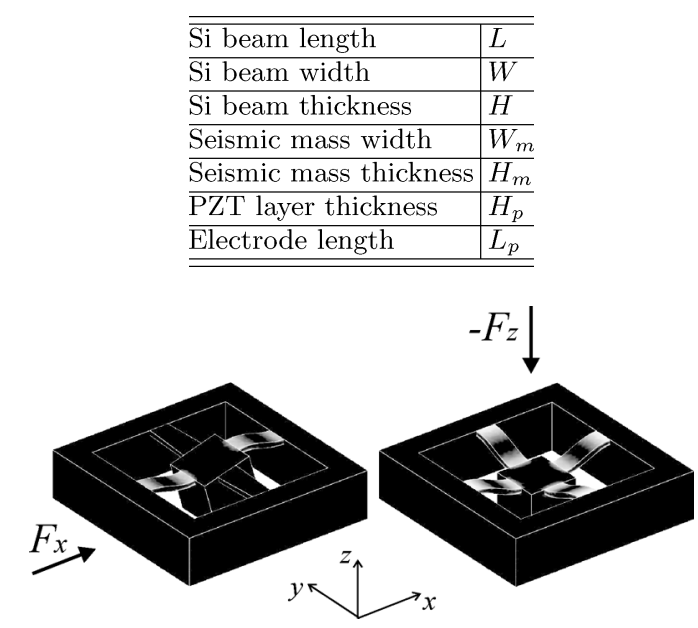

Fig. 3. The figure shows the two modes of operation, a horizontal acceleration (left) and a vertical acceleration (right). The grayscale indicates the strain energy density, $\left[\mathrm{Jm}^{-3}\right]$, in arbitrary units where the bright gray represents a large value.

thickness of the silicon beam, the width and thickness of the seismic mass and, finally, the thickness of the PZT layer, as shown in Fig. 2 and Table I.

The suggested fabrication process flow is based on a backside advanced silicon etching (ASE) of a SOI wafer defining the beam thickness. A deep reactive ion etch (DRIE) releases the seismic mass and defines the four beams. The PZT is screen printed on $\mathrm{SiO}_{2} / \mathrm{Ti} / \mathrm{Pt}$ sandwich structure working as bottom electrode and diffusion barrier layer [11]. Finally, the top electrode is patterned using a standard liftoff process [12].

The accelerometer has two modes of operation, as shown in Fig. 3. For a horizontal acceleration ( $x$ or $y$-direction), the seismic mass will make a rotational movement and the two beams along the direction of the acceleration will have anti-symmetric deflections. The two beams orthogonal to the acceleration will experience torsion and no signal will be generated in these. For a vertical acceleration, all four beams will have a symmetric deflection. As only the two beams along the direction of the horizontal acceleration will give a signal, it is possible to distinguish the three components of the accelerations vector when reading out the signals from the four beams. The normalized output signals from the four beams, $\mathrm{b}_{1}-\mathrm{b}_{4}$, for acceleration in the $x, y$, and $z$-directions are $[+1,0,-1,0],[0,+1,0,-1]$, and $[1,1,1,1]$, respectively, thus the acceleration components may be separated using simple algebra.

The analytical model presented in this paper describes how the sensitivity for the two modes of operation varies as a function of the six independent parameters shown in Table I.

\section{Piezoelectric Material Response}

In the analysis, we shall consider primarily the beam $\mathrm{b}_{1}$ along the $x$ axis shown in Fig. 2. Symmetry will then allow the results to apply also to the other beams.

The mechanical movement of the seismic mass due to acceleration induces a stress, $[\sigma]$, in the PZT layer. The piezoelectric effect in the stressed PZT layer results in an electric field, [E], in the piezoelectric material [13]

$$
[E]=-[g][\sigma]
$$

where a six vector notation is used and $[g]$ is the piezoelectric voltage coefficient matrix with the structure

$$
[g]=\left[\begin{array}{llllll}
0 & 0 & 0 & 0 & g_{15} & 0 \\
0 & 0 & 0 & g_{24} & 0 & 0 \\
g_{31} & g_{32} & g_{33} & 0 & 0 & 0
\end{array}\right] .
$$

For the accelerometer design shown in Fig. 1, we note that only normal stresses, $\sigma_{x}, \sigma_{y}$, and $\sigma_{z}$ may cause an electric field component in the $z$-direction and, thus, contribute to the output signal. The stress $\sigma_{z}$ may be assumed zero since the surface is stress free and the beam is thin, while the axial stress, $\sigma_{x}$, and the transversal stress, $\sigma_{y}$ are both nonzero. The general relation between the normal stresses, $\sigma_{i}$, and normal strains, $\epsilon_{i}$, in all four beams is then

$$
\epsilon_{x}=S_{11} \sigma_{x}+S_{12} \sigma_{y}, \text { and } \epsilon_{y}=S_{22} \sigma_{y}+S_{21} \sigma_{x}
$$

where $S_{i j}$ are compliance coefficients and $\epsilon_{x}$ and $\epsilon_{y}$ are the strain components in the $x$ and $y$-direction, respectively. Most analytical models for beams or cantilevers assume very narrow beams $(L \gg W)$ and that the beam is not constrained in the $y$-direction, such that $\sigma_{y}=0$. In the present accelerometer design this assumption, however, is invalid since the beam length and width are of the same order of magnitude. A better assumption in this case is that the transversal strain in the $y$-direction is zero, $\epsilon_{y}=0$ [7], a condition that usually only apply for thin, wide beams, while it is appropriate here due to the proximity of the frame and seismic mass since the beams are short. [7]. The piezoelectric material and silicon posses sufficient symmetry to ensure $S_{12}=S_{21}$ and $S_{11}=S_{22}$. Using (3) and the condition $\epsilon_{y}=0$, the stresses in the beam $\mathrm{b}_{1}$ thus becomes

$$
\sigma_{x}=\frac{S_{11}}{S_{11}^{2}-S_{12}^{2}} \epsilon_{x} \equiv Y \epsilon_{x}, \text { and } \sigma_{y}=\frac{-S_{12}}{S_{11}^{2}-S_{12}^{2}} \epsilon_{x}
$$

where an effective elastic modulus $Y \equiv S_{11} /\left(S_{11}^{2}-S_{12}^{2}\right)$ has been defined for convenience. The total stress $\sigma_{T}$ is the sum of these stresses

$$
\sigma_{T}=\sigma_{x}+\sigma_{y}=\frac{1}{S_{11}+S_{12}} \epsilon_{x}
$$


The stress in the PZT layer will result in an electric field in the $z$-direction, $E_{z}$, according to (1) and (2). For PZT $g_{31}=g_{32}$ and the electric field is, therefore

$$
E_{z}=-g_{31} \sigma_{T}^{\mathrm{PZT}}=-\frac{g_{31}}{S_{11}^{\mathrm{PZT}}+S_{12}^{\mathrm{PZT}}} \epsilon_{x}
$$

where superscript means that the parameter is valid for the PZT layer. The voltage sensitivity, $S_{V}$ with the unit $\mathrm{Vs}^{2} \mathrm{~m}^{-1}$, is the open circuit potential difference between the top and bottom electrode per acceleration and is found by integration of the electric field across the thickness of the PZT for an infinitely short electrode. For a real electrode with the length $L_{p}$, the effective sensitivity is the mean of the sensitivity across the length of the electrode

$$
\begin{aligned}
S_{V} & =\frac{1}{a L_{p}} \int_{0}^{L_{p}} \int_{0}^{H_{p}}-E_{z} d z d x=\frac{H_{p} g_{31}}{\left(S_{11}^{\mathrm{PZT}}+S_{12}^{\mathrm{PZT}}\right)} \frac{\overline{\epsilon_{x}}}{a} \\
& =H_{p} g_{31} \frac{\overline{\sigma_{T}}}{a}
\end{aligned}
$$

where $a$ is the acceleration, $H_{p}$ is the distance between the top and bottom electrodes as well as the thickness of the PZT layer, while $\overline{\epsilon_{x}}$ is the mean strain and $\overline{\sigma_{T}}$ the mean total stress in the active PZT volume between electrodes.

The capacitance of a set of active electrodes is to a very good approximation obtained from the parallel plate capacitor approximation

$$
C_{\mathrm{PZT}}=\frac{W L_{p}}{H_{p}} \frac{d_{31}}{g_{31}}
$$

where $d_{31} / g_{31}$ is the effective permittivity of the PZT in the $z$-direction, and $d_{31}$ is the piezoelectric charge coefficient of PZT, while $W L_{p}$ is the effective electrode area.

The charge sensitivity, $S_{Q}$ with the unit $C s^{2} m^{-1}$, of the accelerometer is proportional to the voltage sensitivity, $S_{Q}=$ $C_{\mathrm{PZT}} S_{V}$, and is the amount of short circuit charge generated on the electrodes by the active PZT per acceleration

$$
S_{Q}=\frac{W L_{p} d_{31}}{\left(S_{11}^{\mathrm{PZT}}+S_{12}^{\mathrm{PZT}}\right)} \frac{\overline{\epsilon_{x}}}{a} .
$$

High charge sensitivity reduces the effect of stray capacitances on the effective output signal and thus improves the signal-tonoise ratio.

The mean strain in the PZT is different for the two modes of operations, vertical and lateral acceleration, resulting in different sensitivities in the vertical and horizontal direction which is the topic of the following sections.

\section{STATIC ACCELEROMETER RESPONSE}

From (7) it follows that the key problem is to analyze the strain distribution, $\epsilon_{x}$, in the beams when these are deformed due to the acceleration load. In the analysis, we shall ignore the effect of the electrodes on the beam stiffness since they are very thin compared to other material thicknesses. In general, the strain will have two contributions one due to bending, $-z w^{\prime \prime}$ (the double prime signify the second derivative with respect to $x$ ), and one due to stretching, $\epsilon_{x_{N}}$

$$
\epsilon_{x}(x, z)=-z w^{\prime \prime}+\epsilon_{x_{N}}
$$

where $w(x)$ is the deflection of the beam in the $z$-direction; the bending term is valid at small displacements. The deflection is a solution to Euler's static beam equation

$$
\left[\left(Y I_{y}\right)_{\mathrm{eff}} w^{\prime \prime}\right]^{\prime \prime}=-M^{\prime \prime}=0
$$

where $M=-\left(Y I_{y}\right)_{\mathrm{eff}} w^{\prime \prime}$ is the bending moment and $\left(Y I_{y}\right)_{\mathrm{eff}}$ is an effective bending stiffness of the beam

$$
\left(Y I_{y}\right)_{\mathrm{eff}} \equiv W \int_{z_{\mathrm{bot}}}^{z_{\mathrm{top}}} Y z^{2} d z
$$

We shall consider only designs where the neutral plane $z=0$ is at the interface between PZT and silicon such that the strain has the same sign throughout the PZT film; this condition maximize the sensitivity of the accelerometer. In this case, the effective bending stiffness becomes

$$
\begin{aligned}
\left(Y I_{y}\right)_{\mathrm{eff}} & =\frac{W}{3}\left(Y_{\mathrm{PZT}} H_{p}^{3}+Y_{S i} H^{3}\right) \\
& =\frac{W}{3} Y_{S i} H^{3}(1+\gamma)
\end{aligned}
$$

where the parameter $\gamma$ is the ratio of PZT to silicon thicknesses

$$
\gamma \equiv \frac{H_{p}}{H}=\sqrt{\frac{Y_{S i}}{Y_{\mathrm{PZT}}}}
$$

which due to the requirements on the neutral plane position is related to the ratio of effective elastic moduli. With the material parameters used $\gamma \simeq 1$. 42 , while if $S_{12}^{2}$ terms are ignored (corresponds to the $\sigma_{y}=0$ condition, where $Y$ should be replaced by $\left.1 / S_{11}\right) \gamma \simeq 1$. 46 results.

\section{A. Vertical Acceleration $a_{z}$}

An acceleration $a_{z}$ in the $z$-direction causes a net force $F_{a_{z}}=m a_{z}$ on the seismic mass with the mass $m$. The force is evenly shared between the four beams and thus causes a constant shear force $F_{S}=m a_{z} / 4$ in the beam $b_{1}$ which results in a bending strain, while stretching may be ignored due to the small displacement. The boundary conditions for the beam $b_{1}$ are $w(0)=0$, and $w^{\prime}(0)=w^{\prime}(L)=0$ since the beam is rigidly clamped at the frame and due to symmetry. The resulting deflection is the solution to Euler's beam equation, (11), with these boundary conditions

$$
w(x)=\frac{1}{48} \frac{m a_{z}}{\left(Y I_{y}\right)_{\mathrm{eff}}} x^{2}(3 L-2 x)
$$

with the resulting strain distribution, $\epsilon_{x_{v}}$, for vertical acceleration

$$
\epsilon_{x_{v}}=-z w^{\prime \prime}=-\frac{1}{8} \frac{m a_{z}}{\left(Y I_{y}\right)_{\mathrm{eff}}}(L-2 x) z .
$$




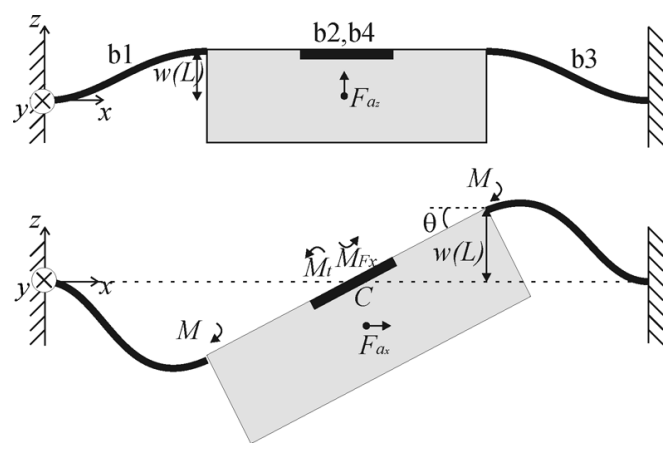

Fig. 4. Cross-sectional view of the vertical deflection (top) and the horizontal deflection (bottom). A moment balance at the point $C$ is used to find to deflection angle $\theta$.

The average strain in the volume between the electrodes per acceleration is thus

$$
\frac{\overline{\epsilon_{x_{v}}}}{a_{z}}=\frac{1}{H_{p} L_{p}} \int_{0}^{L_{p}} \int_{0}^{H_{p}} \frac{\epsilon_{x}}{a_{z}} d z d x=-\frac{1}{16} \frac{m H_{p}\left(L-L_{p}\right)}{\left(Y I_{y}\right)_{\mathrm{eff}}}
$$

the magnitude of which decreases with increasing electrode length, thus the magnitude of the voltage sensitivity decreases with increasing electrode length; the charge sensitivity, however, is maximized for $L_{p}=L / 2$ which is thus the optimal electrode length for vertical acceleration. In that case the average strain in the volume between the electrodes per acceleration is

$$
\frac{\overline{\epsilon_{x_{v}}}}{a_{z}}=-\frac{1}{32} \frac{m H_{p} L}{\left(Y I_{y}\right)_{\mathrm{eff}}} .
$$

\section{B. Horizontal Acceleration $a_{x}$}

An acceleration $a_{x}$ in the $x$-direction causes a net force $F_{a_{x}}=$ $m a_{x}$ on the seismic mass acting on the centre of mass. This force load is equivalent to a combination of a force $F_{a_{x}}$ and a torque $T=-H_{m} F_{a_{x}} / 2=-H_{m} m a_{x} / 2$ around the $y$ axis acting at $C$ in Fig. 4. The torque causes a rotation of the seismic mass with the rotation angle $\theta$, as shown in Fig. 4, with a resulting bending of beams $b_{1}$ and $b_{3}$ and torsion of beams $b_{2}$ and $b_{4}$. The bending contributes to the sensitivity, while the torsion does not since only shear stresses result and $g_{34}=g_{35}=g_{36}=0$. The force causes a uniaxial stretching or compression of beams $b_{1}$ and $b_{3}$ and an in-plane bending of beams $b_{2}$ and $b_{4}$. The uniaxial stretching contributes to the sensitivity, while the in-plane bending does not due to the anti-symmetrical stress distribution.

1) Rotation of the Seismic Mass: The rotation of the seismic mass through the angle $\theta$ causes a displacement of the end of beam $\mathrm{b}_{1}$ of $w(L)=-(1 / 2) W_{m} \sin \theta \simeq-(1 / 2) W_{m} \theta$, while the slope of the end of the beam is $w^{\prime}(L)=\tan \theta \simeq \theta$, as seen in Fig. 4. Here, a first-order Taylor expansion is sufficiently accurate since the angle of rotation is small. An additional displacement of the beam end in the $x$-direction may be ignored since it is in second-order of smallness, $\Delta x \propto \theta^{2}$. With the additional boundary conditions $w(0)=0$ and $w^{\prime}(0)=0$ Euler's beam equation, (11), is solved and the deflection of beam $b_{1}$ becomes

$$
w(x)=\theta\left(\frac{x}{L}\right)^{2}\left[x\left(1+\frac{W_{m}}{L}\right)-L-\frac{3}{2} W_{m}\right]
$$

with the strain distribution $\epsilon_{x_{r}}=-w^{\prime \prime} z$

$$
\epsilon_{x_{r}}=-\frac{z \theta}{L}\left[6 \frac{x}{L}\left(1+\frac{W_{m}}{L}\right)-2-3 \frac{W_{m}}{L}\right]
$$

and the resulting average strain between the electrodes in the case of electrode length $L_{p}=L / 2$ becomes

$$
\overline{\epsilon_{x_{r}}}=\frac{1}{4} \frac{H_{p} \theta}{L}\left(1+3 \frac{W_{m}}{L}\right) .
$$

The bending moment associated with the beam bending is $M(x)=-w^{\prime \prime}(x)\left(Y I_{y}\right)_{\mathrm{eff}}$

$$
M(x)=-\frac{\theta}{L}\left[6 \frac{x}{L}\left(1+\frac{W_{m}}{L}\right)-2-3 \frac{W_{m}}{L}\right]\left(Y I_{y}\right)_{\mathrm{eff}} .
$$

This equation is valid in the beam, but also in the seismic mass until the point $C$ where the bending moment in the beam approximation becomes discontinuous. Just to the left of point $C$, the bending moment becomes

$$
M_{C}=-\frac{\theta}{L}\left[4+6 \frac{W_{m}}{L}+3\left(\frac{W_{m}}{L}\right)^{2}\right]\left(Y I_{y}\right)_{\mathrm{eff}}
$$

where, for simplicity, we define $M_{C} \equiv-K_{C} \theta / L$. The torque balance at point $C$ in Fig. 4 is

$$
T=-2 M_{t}+2 M_{C}=-2\left(K_{C}+K_{\theta}\right) \frac{\theta}{L}
$$

where $M_{t}$ is the torque due to torsion of beam $\mathrm{b}_{2}$ or $\mathrm{b}_{4}$ with $M_{t}=K_{\theta} \theta / L$, where $K_{\theta}$ is the torsional rigidity of the beams [14]. It follows that the rotation angle per acceleration becomes

$$
\begin{aligned}
\frac{\theta}{a_{x}} & =-\frac{T L}{2\left(K_{C}+K_{\theta}\right) a_{x}}=\frac{H_{m} m L}{4\left(K_{C}+K_{\theta}\right)} \\
& =\frac{H_{m} m L}{4\left(Y I_{y}\right)_{\text {eff }}} \frac{1}{4+6 \frac{W_{m}}{L}+3\left(\frac{W_{m}}{L}\right)^{2}+\frac{K_{\theta}}{\left(Y I_{y}\right)_{\text {eff }}}} .
\end{aligned}
$$

When this equation is inserted in (21), the average strain between the electrodes per acceleration due to bending becomes

$$
\frac{\overline{\epsilon_{x_{r}}}}{a_{x}}=\frac{H_{p} H_{m} m}{16\left(Y I_{y}\right)_{\mathrm{eff}}} \frac{\left(1+3 \frac{W_{m}}{L}\right)}{4+6 \frac{W_{m}}{L}+3\left(\frac{W_{m}}{L}\right)^{2}+\frac{K_{\theta}}{\left(Y I_{y}\right)_{\text {eff }}}} .
$$

If the beam width is large compared to the total beam thickness, $W \gg H+H_{p}$, the torsional rigidity, $K_{\theta}$, is to a good approximation

$$
K_{\theta}=\frac{1}{3} \frac{W H^{3}}{S_{44}^{\mathrm{Si}}}\left(1+\frac{S_{44}^{\mathrm{Si}} H_{p}^{3}}{S_{44}^{\mathrm{PZT}} H^{3}}+3 \frac{\left(\frac{H_{p}}{H}+1\right)^{2}}{1+\frac{S_{44}^{\mathrm{PZT}} H}{S_{44}^{\mathrm{Si}_{p}}}}\right)
$$

as proven by an approximate solution to Prandtl's torsion stress function equation, $\nabla_{2 D}^{2} \Phi=-2 G \theta / L$, where $G$ is the shear modulus and $\Phi$ is the stress function, which is zero on the 


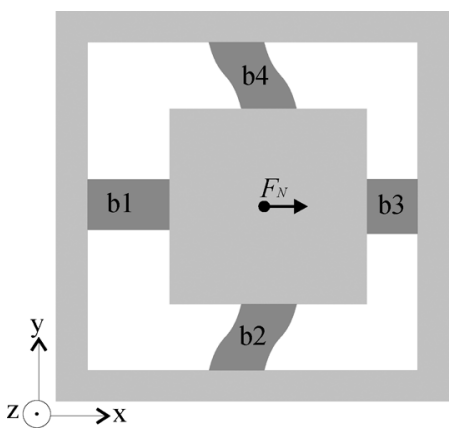

Fig. 5. Top view of the seismic mass and the four beams deflected due to an horizontal acceleration. This movement results in a lateral force $F_{N}$. The beams $b_{2}$ and $b_{4}$ are bending, beam $b_{1}$ is stretched, and beam $b_{3}$ is compressed.

boundary of the beam [14]; the approximate solution has to fulfil the additional conditions that the strain and the stress function are continuous across the material boundary. The torsion moment is then $M_{t}=2 \iint \Phi d z d y$ [14]. Here, the shear moduli are $G_{S i}=1 / S_{44}^{\mathrm{Si}}$ and $G_{\mathrm{PZT}}=1 / S_{44}^{\mathrm{PZT}}=1 / S_{55}^{\mathrm{PZT}}$. The ratio $K_{\theta} /\left(Y I_{y}\right)_{\text {eff }} \equiv \alpha$ then becomes

$$
\alpha=\frac{1}{S_{44}^{\mathrm{Si}} Y_{S i}(1+\gamma)}\left(1+\frac{S_{44}^{\mathrm{Si}}}{S_{44}^{\mathrm{PZT}}} \gamma^{3}+3 \frac{(\gamma+1)^{2}}{1+\frac{S_{44}^{\mathrm{PZT}}}{\gamma S_{44}^{\mathrm{Si}}}}\right)
$$

with the material parameters used here $\alpha \simeq 1.3$.

2) Lateral Force: The force, $F_{a_{x}}$, due to acceleration in the $x$-direction causes a lateral force $F_{N}$, as seen in Fig. 5, a normal stress $\sigma_{x_{N}}$, and uniform strain $\epsilon_{x_{N}}$ in the beam $\mathrm{b}_{1}$. The lateral force is integrally related to the stress

$$
\begin{aligned}
F_{N} & =W \int_{-H}^{H_{p}} \sigma_{x_{N}} d z=W\left(Y_{S i} H+Y_{\mathrm{PZT}} H_{p}\right) \epsilon_{x_{N}} \\
& =W H Y_{S i}\left(1+\frac{1}{\gamma}\right) \epsilon_{x_{N}} \equiv K_{N} L \epsilon_{x_{N}}
\end{aligned}
$$

where the spring constant for elongation $K_{N}$ has been defined for convenience. In-plane bending of the beams $b_{2}$ or $b_{4}$ is characterized by a spring constant

$$
K_{B}=\left(\frac{W}{L}\right)^{3} Y_{S i} H\left(1+\frac{1}{\gamma}\right)
$$

With these definitions the lateral force balance becomes $m a_{x}=$ $\left(2 K_{N}+2 K_{B}\right) L \epsilon_{x_{N}}$ and thus the strain per acceleration is

$$
\begin{aligned}
\frac{\epsilon_{x_{N}}}{a_{x}} & =\frac{m}{2 L\left(K_{N}+K_{B}\right)} \\
& =\frac{1}{Y_{S i}} \frac{m}{2 L H\left(1+\frac{1}{\gamma}\right)\left[\frac{W}{L}+\left(\frac{W}{L}\right)^{3}\right]}
\end{aligned}
$$

which by use of the definition of the effective bending stiffness $\left(Y I_{y}\right)_{\mathrm{eff}}(13)$ is simplified to

$$
\frac{\epsilon_{x_{N}}}{a_{x}}=\frac{m}{6\left(Y I_{y}\right)_{\mathrm{eff}}} \frac{\gamma H^{2}}{\left[1+\left(\frac{W}{L}\right)^{2}\right]}
$$

for easy comparison to the strain due to bending, see (26).
3) Sensitivity Ratio: The total average strain per acceleration between the electrodes is obtained by adding (26) and (33)

$$
\frac{\overline{\epsilon_{x_{h}}}}{a_{x}}=\frac{m \gamma H L}{32\left(Y I_{y}\right)_{\mathrm{eff}}} \times \Gamma=-\frac{\overline{\epsilon_{x_{v}}}}{a_{z}} \times \Gamma
$$

where the strain per acceleration has been written in terms of the strain per acceleration for vertical acceleration, (18), times the sensitivity ratio $\Gamma$, the ratio of the horizontal acceleration sensitivity $S_{V h}$ to the vertical acceleration sensitivity $S_{V v}$

$$
\Gamma \equiv \frac{\left|S_{V h}\right|}{\left|S_{V v}\right|}=\frac{32}{6} \frac{\frac{H}{L}}{1+\frac{W^{2}}{L^{2}}}+\frac{2 \frac{H_{m}}{L}\left(1+3 \frac{W_{m}}{L}\right)}{4+6 \frac{W_{m}}{L}+3 \frac{W_{m}^{2}}{L^{2}}+\alpha} .
$$

This ratio should preferably be close to one, and it is seen to be dependent on all accelerometer geometries.

\section{Sensitivities $S_{V}$}

The absolute voltage sensitivity to vertical acceleration, $S_{V v}$, is easily obtained by combining (7) and (18)

$$
S_{V v}=-\frac{1}{32} \frac{\gamma^{2} g_{31} m H^{2} L}{\left(S_{11}^{\mathrm{PZT}}+S_{12}^{\mathrm{PZT}}\right)\left(Y I_{y}\right)_{\mathrm{eff}}} .
$$

Using the definition of the effective bending stiffness $\left(Y I_{y}\right)_{\text {eff }}$ (13) and $\gamma$ (14) the expression for the voltage sensitivity may be expressed in terms of PZT material parameters and an accelerometer geometry factor

$$
S_{V v}=-\frac{3}{32} g_{31}\left[\frac{S_{11}-S_{12}}{S_{11}}\right]_{\mathrm{PZT}} \frac{L m}{W H(1+\gamma)} .
$$

It follows that high sensitivity requires a large seismic mass and long beams with a small cross-sectional area. The corresponding equation for the alternative simplifying beam analysis assumption, $\sigma_{y}=0$ which is valid for a narrow beam, becomes

$$
S_{V v}^{\sigma_{y}=0}=-\frac{3}{32} g_{31} \frac{L m}{W H(1+\gamma)}
$$

which is $25 \%$ smaller for the same nominal geometry with the PZT material parameters used here.

The sensitivity to horizontal acceleration is

$$
S_{V h}=-\Gamma S_{V v}
$$

with the sensitivity ratio obtained from (35).

To complete the analysis, the voltage sensitivities to angular acceleration around the coordinate axes are briefly discussed below. Angular acceleration around the $z$ axis causes an in-plane bending of the beams, thus the output voltage and the voltage sensitivity are both zero. Angular acceleration around the $x$ or $y$ axis results in output signals that cannot be distinguished from horizontal acceleration in the $y$ - or $x$-direction, respectively. Due to symmetry the voltage sensitivities are equal for angular acceleration around the $x$ and $y$ axes. Angular acceleration, $\ddot{\varphi}$, around the $x$ axis causes a torque $T=J \ddot{\varphi}$, where $J$ is the moment of inertia of the seismic mass with respect to the $x$ axis through point $C$ in Fig. 4. It follows that the voltage sensitivity to angular acceleration, $S_{V \varphi}$, is proportional 
to the part of the voltage sensitivity for horizontal acceleration, $S_{V h}^{r}$, that is due to rotation of the seismic mass (i.e., the part originating from (26))

$$
S_{V \varphi}=S_{V h}^{r} \frac{2 J}{m H_{m}}=S_{V h}^{r}\left(\frac{1}{6} \frac{W_{m}^{2}}{H_{m}}+\frac{2}{3} H_{m}\right) .
$$

For a fixed $W_{m}$ the term in the parenthesis has a broad minimum at $H_{m}=W_{m} / 2$; it follows, that designs that approximately fulfil that relation have the least relative sensitivity to angular acceleration. We shall not discuss angular acceleration further.

\section{RESONANT FREQUENCY}

Generally, the frequency response of sensitivity of accelerometer is flat in the range below its resonance and shows a sharp peak at the resonance and diminishes towards almost zero above the resonance. Therefore, the operational bandwidth is limited by the lowest resonant frequency. Accelerometers with comparable sensitivities will also have comparable resonant frequencies for the two modes. An approximate expression for the angular resonant frequency for the vertical mode $\omega_{v}$ is quite simple [15]

$$
\omega_{v}=\sqrt{\frac{K}{m}}, \text { with } K=48 \frac{\left(Y I_{y}\right)_{\mathrm{eff}}}{L^{3}}
$$

and this expression is used as a design tool in the discussion section. By use of the effective bending stiffness, (13), the angular resonant frequency becomes

$$
\omega_{v}=4 \sqrt{\frac{W H^{3}(1+\gamma) Y_{S i}}{\varrho L^{3} H_{m} W_{m}^{2}}} .
$$

where $m=\varrho_{S i} H_{m} W_{m}^{2}$ has been used. Using (36) and (42), a sensitivity bandwidth relation may be expressed as

$$
\left|S_{V v}\right| \omega_{v}^{2}=\frac{3}{2} \frac{g_{31}}{\left(S_{11}^{\mathrm{PZT}}+S_{12}^{\mathrm{PZT}}\right)} \frac{H_{p}^{2}}{L^{2}}
$$

The similar expression for the charge sensitivity bandwidth relation becomes

$$
\left|S_{Q v}\right| \omega_{v}^{2}=\frac{3}{4} \frac{d_{31}}{\left(S_{11}^{\mathrm{PZT}}+S_{12}^{\mathrm{PZT}}\right)} \frac{W H_{p}}{L} .
$$

It follows that the active PZT volume should be maximized in order to get high charge-sensitivity bandwidth.

The expression for the resonant frequency of the mode corresponding to lateral acceleration is quite complicated; an approximate voltage sensitivity bandwidth relation, however, becomes quite simple if the normal force contribution to the sensitivity in the interest of simplicity is ignored

$$
\left|S_{V_{h}}\right| \omega_{h}^{2} \gtrsim\left|S_{V_{v}}\right| \omega_{v}^{2} \times \frac{H_{m}\left(L+3 W_{m}\right)}{4 H_{m}^{2}+W_{m}^{2}} \equiv\left|S_{V_{v}}\right| \omega_{v}^{2} \times \Psi
$$

where $\omega_{h}$ is the angular resonant frequency for the horizontal mode of operation, and the ratio $\Psi$ has been defined for convenience. This relation may be used for optimization if it is essential that sensitivities and bandwidths both are matched for the two modes of operation, this is however only possible for rather long beams and wide seismic masses compared to the thickness of the seismic mass; in that limit, however, modified sensitivity relations are necessary due to the long beam. For shorter beams, however, $\left|S_{V_{h}}\right| \omega_{h}^{2}<\left|S_{V_{v}}\right| \omega_{v}^{2}$. In the very short beam limit, $L \ll W_{m}$, the ratio $\Psi$ is maximized for $W_{m} \simeq 2 H_{m}$ and assumes the value $\Psi=3 / 4$; corresponding to a horizontal mode resonant frequency which is approximately $15 \%$ less than the vertical mode resonant frequency if the sensitivities are matched.

\section{FEM MODEL}

A 3-D COMSOL FEM model is used to verify the analytical model. The FEM model takes all the coefficients of the material matrixes into account whereas the analytical model is based on several simplifying assumptions. The FEM model includes many mesh points in the vertical $z$-direction of the beams in order to accurately model the torsion and bending of the beams for horizontal accelerations.

In the real accelerometer not only the beams but also the fixed frame will be stressed. This effect is not included in the analytical model since solid clamped boundary conditions are used, while the flexibility of frame is included in the FEM model, where only the position of the outer rim of the frame is fixed. The absolute sensitivity is affected by the finite stiffness of the frame, while the sensitivity ratio is unaffected as verified by FEM. The analytical model is, therefore, still quite good even without an accurate modeling of the frame.

\section{NUMERICAL EVALUATION}

The material coefficients used for PZT in the numerical evaluation are those for the TF26 thick-film provided by InSensor A/S, while for the Si substrate standard coefficients for a $\langle 100\rangle$ silicon wafer are used. All values of the material coefficients are included in Appendix A. The piezoelectric coefficients are those of a screen printed PZT film.

The full parameter space consists of six independent dimensions, as shown in Table I. The PZT thickness, however, is assumed related to the silicon beam thickness according to (14). The thickness of the seismic mass is limited by the wafer thickness and in the numerical evaluation it is fixed to $H_{m}=1000 \mu \mathrm{m}$. This essentially leaves four free design parameters: beam thickness, beam width, beam length and mass width.

In the numerical evaluation, the analytical model is verified using the FEM model in the mass width and beam length space, while the beam thickness and beam width are fixed. All parameters are shown in Table II.

\section{A. Strain Evaluation}

Figs. 6 and 7 show comparisons of the mean strain in the $x$-direction for vertical and horizontal accelerations between the analytical model, (18) and (34), respectively, and the FEM model. Good agreement between the analytical and the numerical models is seen, it follows that the mean strain in the deflected beams can be estimated from the analytical model with a reasonable accuracy in the given parameter space. 
TABLE II

GeOMeTRICAL PARAMETERS USED IN THE FEM MODEL AND REDUCED MATERIAL PARAMETERS USED FOR NUMERICAL EVALUATION OF THE ANALYTICAL MODEL

\begin{tabular}{c|c|c|c|c}
\hline \hline$H_{m}$ & $H$ & $H_{p}$ & $W$ & \\
\hline$[\mu \mathrm{m}]$ & {$[\mu \mathrm{m}]$} & {$[\mu \mathrm{m}]$} & {$[\mu \mathrm{m}]$} & \\
\hline 1000 & 10 & 14.5 & 300 & \\
\hline \hline$\varrho_{\mathrm{Si}}$ & $Y_{\mathrm{Si}}$ & $Y_{\mathrm{PZT}}$ & $\gamma$ & $\alpha$ \\
\hline$\left[\mathrm{kgm}^{-3}\right]$ & {$[\mathrm{GPa}]$} & {$[\mathrm{GPa}]$} & & \\
\hline 2330 & 142.5 & 70.2 & 1.42 & 1.30 \\
\hline \hline
\end{tabular}

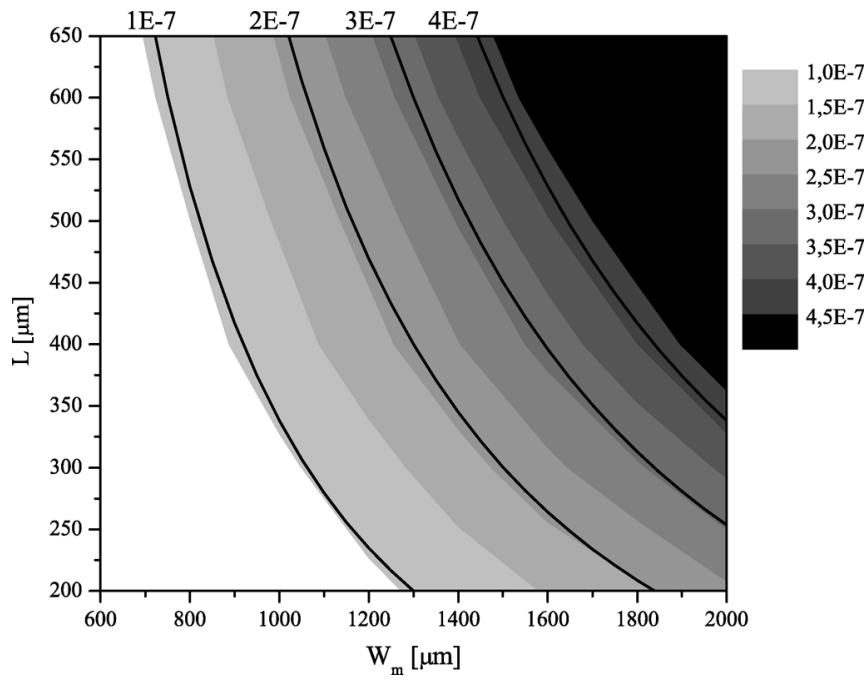

Fig. 6. The mean strain, $\overline{\epsilon_{x_{v}}}$, for accelerations in the vertical direction as a function of seismic mass width $W_{m}$, and beam length $L$. The filled contour plot are the FEM model results, while the black lines are the analytical model results. Increasing beam length and increasing mass width increases the mean strain.

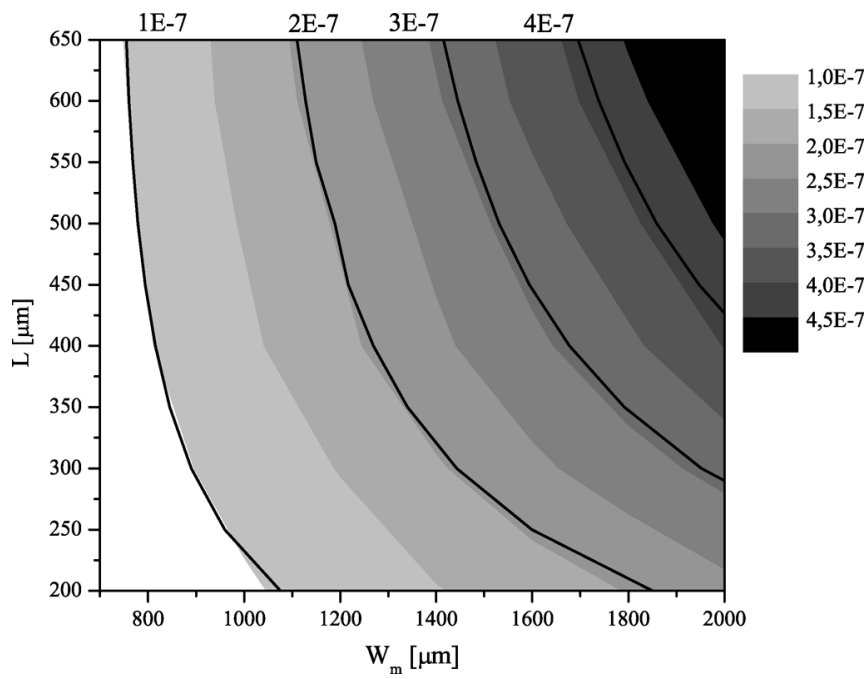

Fig. 7. The mean strain, $\overline{\epsilon_{x_{b}}}$, for horizontal accelerations as a function of mass width $W_{m}$, and beam length $L$. The filled contour plot are the FEM model results, while the full lines are the analytical model results. Increasing beam length and increasing mass width increases the mean strain.

According to the analytical model the relative sensitivity, (35), is equal to the ratio between mean strains induced by horizontal and vertical accelerations. Fig. 8 shows the ratio between the two mean strains, $\overline{\epsilon_{x, h}} / \overline{\epsilon_{x, v}}$, found using the FEM model and the curve corresponding a strain ratio $\Gamma=1$ calculated from the analytical model (35). The two models are

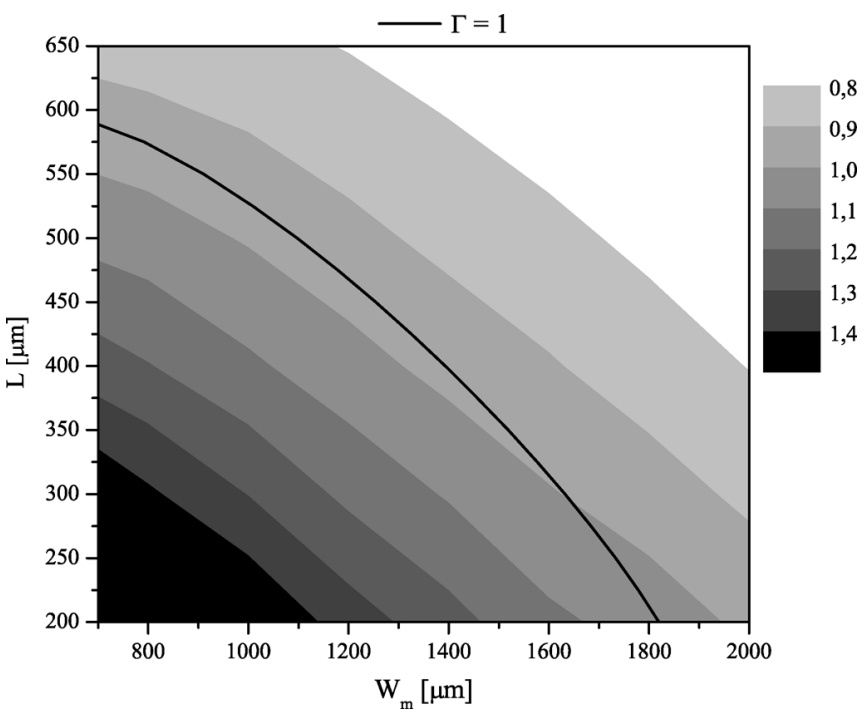

Fig. 8. The ratio between the mean strains, $\overline{\varepsilon_{x_{h}}} / \overline{\varepsilon_{x_{v}}}$, plotted in Figs. 6 and 7 , respectively, is shown in this figure. The filled contour plot is the FEM model and the black line is based on the analytical model. The relative sensitivity is equal to one along the black line according to the analytical model and again there is good agreement with the FEM model.

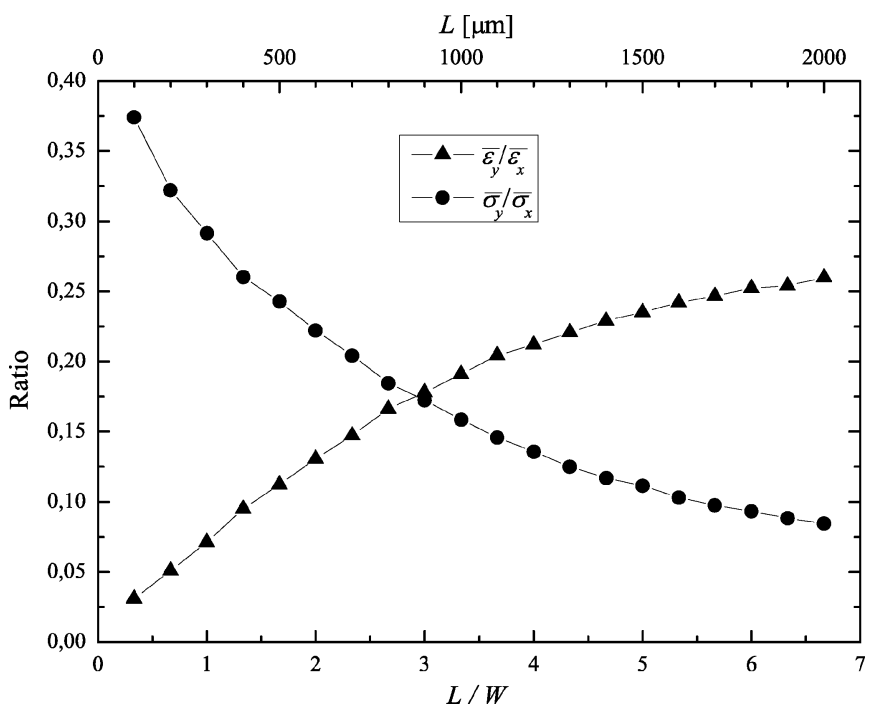

Fig. 9. The ratios $\overline{\epsilon_{y}} / \overline{\epsilon_{x}}$ and $\overline{\sigma_{y}} / \overline{\sigma_{x}}$ as a function of the beam length-width ratio, $L / W$, calculated using the FEM model ( $W=300 \mu \mathrm{m}$ is fixed and $L$ is varied from $100 \mu \mathrm{m}$ to $2000 \mu \mathrm{m}$, the other parameters are shown in Table II).

in quite good agreement, the relative difference is less than 5\%. The strain ratio is seen to decrease with increasing beam length and with increasing seismic mass width.

\section{B. Total Stress Evaluation}

The sensitivity of the accelerometer is linearly proportional to the total mean stress $\bar{\sigma}_{T}$ in the active PZT volume (7). The analytical model is based on a simplified relation between stress and strain, therefore, it is not enough to evaluate the analytical model by comparing the mean strains so in this section the total mean stresses found with the analytical and the FEM model are compared.

The validity of the assumption of zero transversal strain $\epsilon_{y}=$ 0 used in the analytical model is tested by plotting the ratio of mean transversal to longitudinal strain $\overline{\epsilon_{y}} / \overline{\epsilon_{x}}$, and stress $\overline{\sigma_{y}} / \overline{\sigma_{x}}$ 


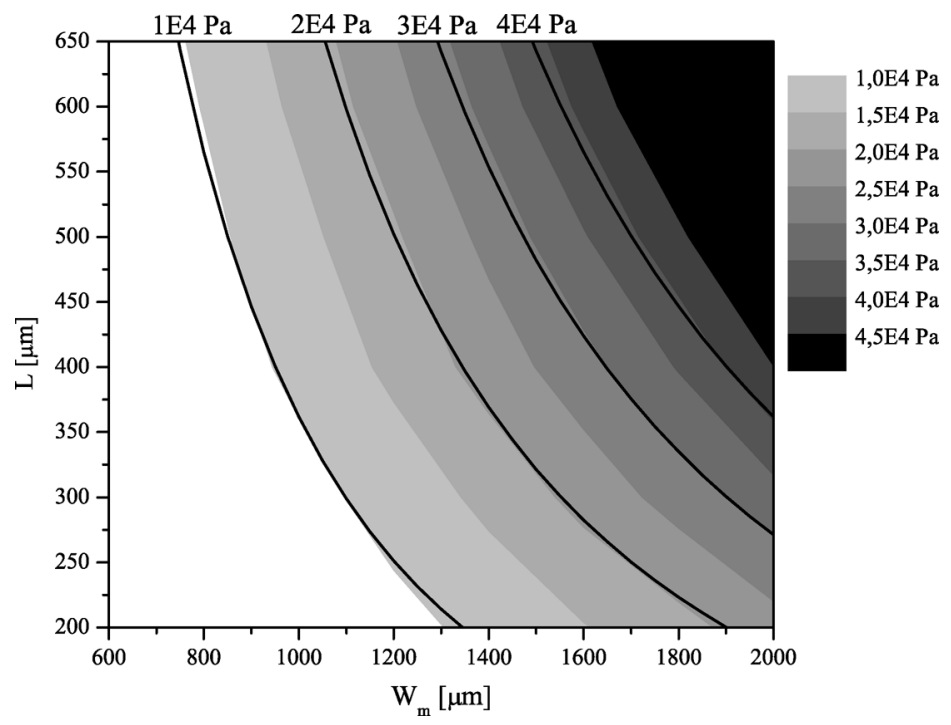

Fig. 10. The total mean stress, $\overline{\sigma_{T v}}$, in the PZT on the beam for vertical accelerations. The full lines are results from the analytical model. The agreement is seen to be excellent.

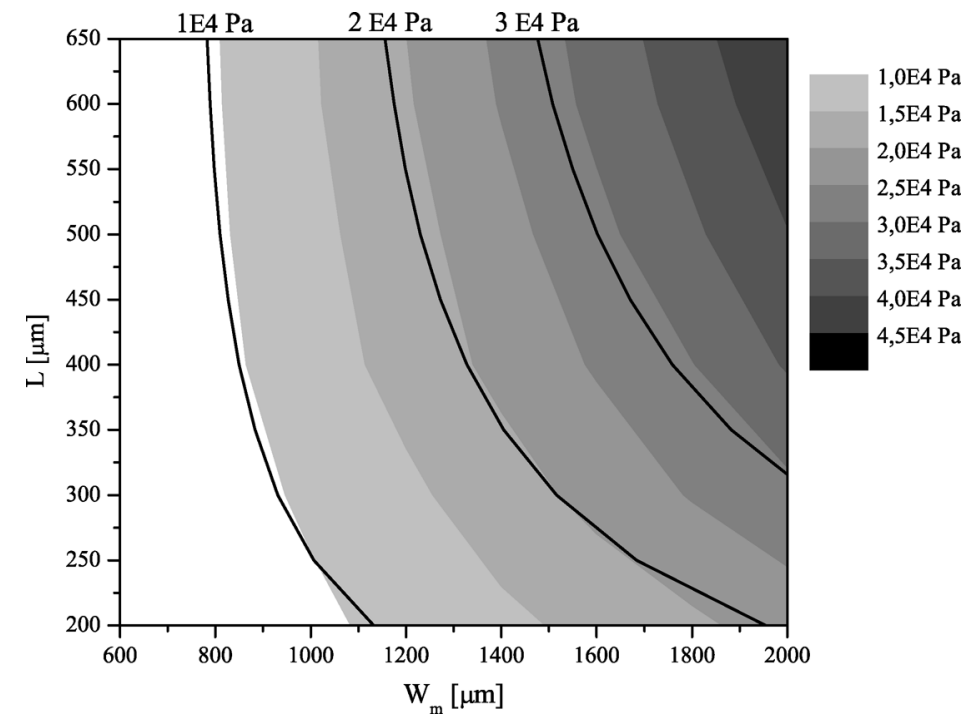

Fig. 11. The total mean stress, $\overline{\sigma_{T_{h}}}$, in the PZT on the beam for horizontal accelerations. The full lines are results from the analytical model. Good agreement between the FEM model and the analytical model is seen, for longer beams the agreement becomes less good.

as a function of the beam length to width ratio $L / W$ as calculated from the numerical FEM model in Fig. 9. The plots in Fig. 9 shows the expected result that in the short beam limit, zero transverse strain is a quite good assumption, while in the long beam limit, zero transverse stress is the better assumption. Within the parameter space relevant to this work, zero transverse strain is the better assumption since the beam length to width ratio $L / W$ is small.

Figs. 10 and 11 show the total mean stress for vertical $\overline{\sigma_{T_{v}}}$ and horizontal $\overline{\sigma_{T_{h}}}$ accelerations, respectively. The total mean stress is in the analytical model given by (5) with the strains obtained from (18) and (34), respectively. Again, a quite good agreement between the analytical model and the FEM model is seen. The agreement between the analytical and FEM model becomes less good for long beams where the beam length-width ratio increases and the effect of a nonzero transversal strain becomes more notable. The stress ratio, $\overline{\sigma_{T_{h}}} / \overline{\sigma_{T_{v}}}$, resulting from the FEM model, as shown in Fig. 12, however, is in very good agreement with the analytical result (35) as the contour $\Gamma=1$ shows. In the parameter range used, the relative error on the sensitivity ratio is less than $3 \%$.

In conclusion, the analytical expression for the sensitivity ratio (35) is very accurate, and even the absolute sensitivities are accurately modeled by (36) and (39) as demonstrated by the, in general, excellent agreement between the results from the analytical model and the FEM model seen in Figs. 10-12.

\section{DISCUSSION}

\section{A. Bandwidth}

The bandwidth is a very important accelerometer parameter. For a triaxial accelerometer, the useful bandwidth is limited by the lowest resonant frequency of the device. Preferably, the resonant frequencies should be identical for all modes of operation; 


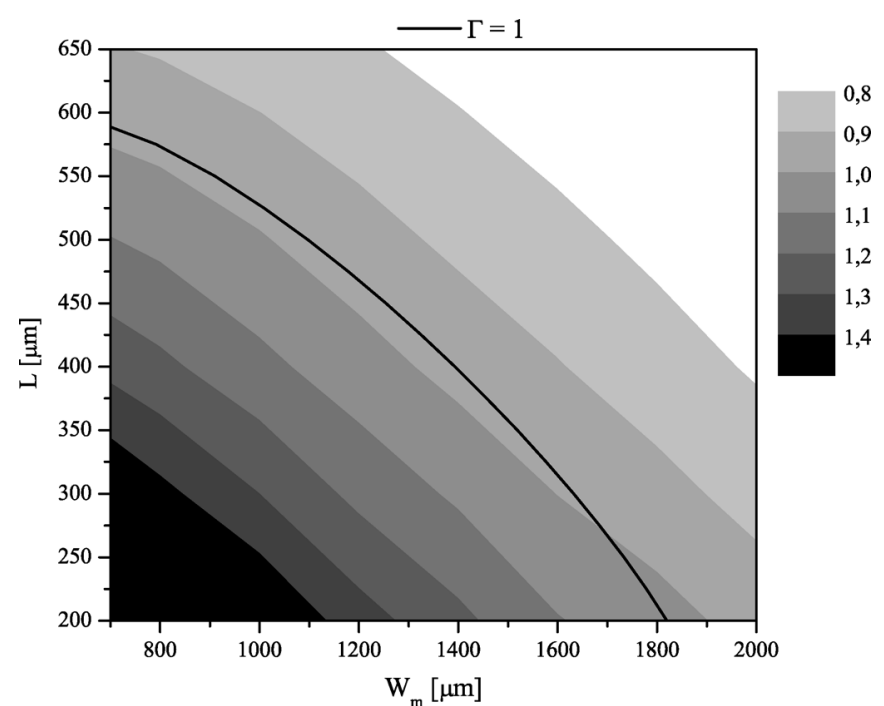

Fig. 12. The ratio, $\overline{\sigma_{T_{h}}} / \overline{\sigma_{T_{v}}}$, between the total mean stresses for vertical and horizontal accelerations. The full line is the contour $\overline{\sigma_{T_{h}}} / \overline{\sigma_{T_{v}}}=1$ according to the analytical model. As in Fig. 8, there is a very good agreement between the analytical model and the FEM model.

small differences between the resonant frequencies, however, are rather unimportant, while an accurate matching of the sensitivities is essential. In a design process where matched sensitivities are ensured by $\Gamma=1$, sufficient resonant frequency match is achieved by allowing $0.75<\Psi<1.25$ as calculated from (45).

The angular resonant frequencies for horizontal accelerations $\omega_{h}$ are due to symmetry the same in the $x$ and $y$-direction. Fig. 13 shows the ratio between the resonant frequencies, $\omega_{v} / \omega_{h}$, calculated from the FEM model for variations in beam length and seismic mass width. For comparison, the contour of matched sensitivity, $\Gamma=1$, according to (35) is shown as a full line. In addition, estimates of the resonant frequency ratio $\sqrt{\Psi / \Gamma}=1$ according to (35) and (45) are shown as a contour line which agrees quite well with the results from the FEM model.

\section{B. Cross Axis Sensitivity}

For horizontal accelerations in the $x$-direction only the beams $b_{1}$ and $b_{3}$ should produce an output signal. Finite output signals from the beams $b_{2}$ and $b_{4}$ cause an undesirable cross-axis sensitivity which degrade the quality of the accelerometer. In the analytical model the cross-axis sensitivity is assumed to be zero due to symmetry of the deflection and since torsion of a beam is not expected to produce an output signal.

Table III shows values for all mean strains and stresses in the beams $\mathrm{b}_{1}$ and $\mathrm{b}_{2}$ calculated for a $1 \mathrm{~g}$ acceleration in the $x$-direction using the FEM model and the corresponding values calculated from the analytical model in cases where expressions are derived. Due to symmetry, the values for the beams $b_{1}$ and $\mathrm{b}_{3}$ are equal in magnitude but of opposite sign, while values for the beams $b_{2}$ and $b_{4}$ are equal. In Table III we note that the normal strain $\overline{\epsilon_{x}}$ and normal stresses $\overline{\sigma_{x}}$ and $\overline{\sigma_{y}}$ are quite accurately modeled, while the assumption of zero normal stress in the $z$-direction, $\overline{\sigma_{z}}=0$, is not exactly fulfilled, the magnitude

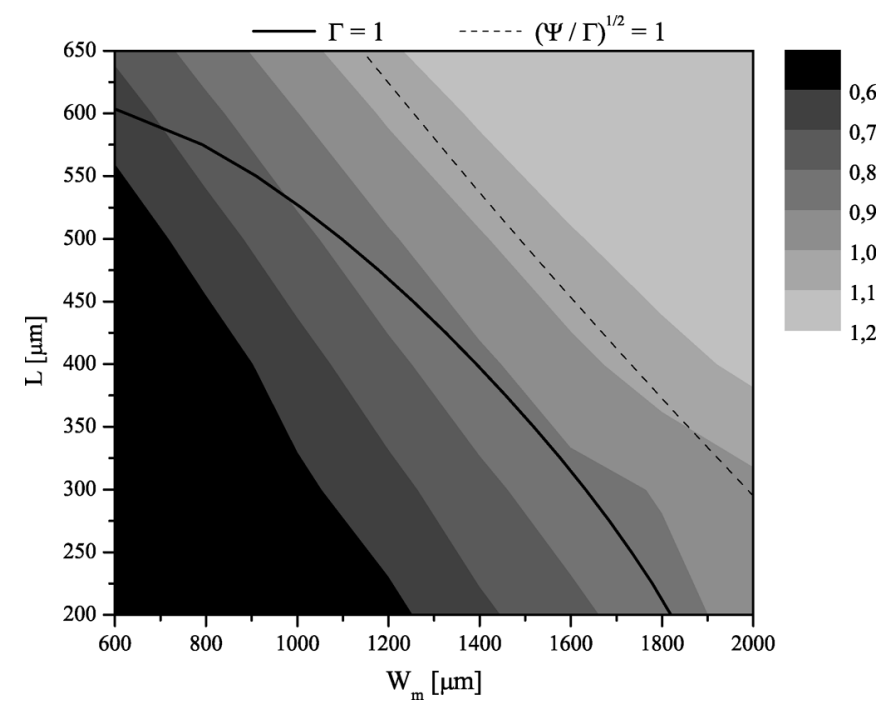

Fig. 13. The contours show the ratio $\omega_{h} / \omega_{v}$, between the horizontal resonant frequency and the vertical resonant frequency calculated using the FEM model. The contour of relative sensitivity equal to one (full line) is shown for comparison. The dashed line is the contour $\sqrt{\Psi / \Gamma}$.

TABLE III

The Table Shows the Mean Normal Stresses and Mean NORMal Strains as Well as the SHEAR StRESS AND STRAIN COMPONENTS Found FROM THE ANALYTICAL MODEL AND THE FEM Model, Respectively. The Values are CAlculated FOR A $1 \mathrm{~g}$ ACCELERATION IN THE $x$-DIRECTION. ( $L=450 \mu \mathrm{m}, W=300 \mu \mathrm{m}, H=10 \mu \mathrm{m}$, $\left.H_{p}=14.2 \mu \mathrm{m}, H_{m}=1000 \mu \mathrm{m}, W_{m}=1000 \mu \mathrm{m}\right)$

\begin{tabular}{c|r|r|r|r|r}
\hline \hline & $\mathrm{b}_{1_{\mathrm{FEM}}}$ & $\mathrm{b}_{1_{\mathrm{Ana}}}$ & $\mathrm{b}_{2_{\mathrm{FEM}}}$ & $\mathrm{b}_{2_{\mathrm{Ana}}}$ & \\
\hline$\overline{\overline{\epsilon_{x}}}$ & 1.4684 & 1.4654 & -0.0036 & 0 & $\times 10^{-7}$ \\
$\overline{\overline{\epsilon_{y}}}$ & -0.1291 & 0 & 0.0011 & 0 & $\times 10^{-7}$ \\
$\overline{\overline{\epsilon_{z}}}$ & -0.8459 & - & 0.0005 & 0 & $\times 10^{-7}$ \\
$\overline{\overline{\epsilon_{x y}}}$ & -0.0001 & - & -0.3634 & - & $\times 10^{-7}$ \\
$\overline{\overline{\epsilon_{y z}}}$ & 0.0004 & - & -0.0001 & - & $\times 10^{-7}$ \\
$\overline{\overline{\epsilon_{x z}}}$ & -0.2056 & - & -0.0137 & - & $\times 10^{-7}$ \\
$\overline{\overline{\overline{\sigma_{x}}}}$ & 10250.8 & 10287.4 & -28.5 & 0 & {$[\mathrm{~Pa}]$} \\
$\overline{\overline{\sigma_{y}}}$ & 2790.4 & 3407.7 & -6.6 & 0 & {$[\mathrm{~Pa}]$} \\
$\overline{\overline{\sigma_{z}}}$ & 423.7 & 0 & 8.8 & 0 & {$[\mathrm{~Pa}]$} \\
$\overline{\overline{\sigma_{x y}}}$ & 0.3 & - & -1213.9 & - & {$[\mathrm{Pa}]$} \\
\hline$\overline{\overline{\sigma_{y z}}}$ & 0.7 & - & -2.4 & - & {$[\mathrm{Pa}]$} \\
$\overline{\overline{\sigma_{x z}}}$ & -966.3 & - & -64.5 & - & {$[\mathrm{Pa}]$} \\
\hline \hline
\end{tabular}

of $\overline{\sigma_{z}}$ is however only $\sim 4 \%$ of the magnitude of $\overline{\sigma_{x}}$; the nonzero value of $\overline{\sigma_{z}}$ is due to the short beams used here, hence, the proximity of the frame restricts deformation of the beam in the $z$-direction. The assumption of zero transverse strain $\overline{\epsilon_{y}}=0$ is not too accurate, the actual magnitude of $\overline{\epsilon_{y}}$ is $\sim 9 \%$ of magnitude of $\overline{\epsilon_{x}}$. Equations are not given for the shear stresses, the nonzero value of the shear stress $\overline{\sigma_{x z}}$ and the corresponding shear strain in beam $b_{1}$, however, is consistent with the shear force acting on the beam, while the nonzero values of shear stress $\overline{\sigma_{x y}}$ and corresponding strain in the beam $b_{2}$ is consistent with the torsional deformation of that beam, while the other shear stress $\overline{\sigma_{y z}}$ due to torsion average to zero. Finally, the shear stress $\overline{\sigma_{x z}}$ in the beam $b_{2}$ is due to the shear force in the beam related to the normal force displacement. Since the shear stresses do not contribute to the output signal the finite value of these stresses are unimportant. The finite value of $\overline{\sigma_{z}}$, however, does contribute to 
the output signal and adds to model errors, and since $g_{33}$ is approximately four times larger in magnitude than $g_{31}$ this could very well be the major error contribution. The cross axis sensitivity is found by comparing the total voltage in the $z$-direction in $\mathrm{b}_{1}$ and $\mathrm{b}_{2},\left(-[g][\bar{\sigma}]_{\mathrm{b} 2}\right)_{z} /\left(-[g][\bar{\sigma}]_{\mathrm{b} 1}\right)_{z}$, which is acceptable low $\sim 0.7 \%$.

\section{Design Strategy}

The sensitivity, the relative sensitivity and the resonant frequency are all functions of the six independent parameters. In the following, we demonstrate how the parameter space is reduced to two independent parameters by introducing relevant restrictions. It should be noted that the two free parameters could be any of the six starting parameters and the discussion here is only an example of how the six dimensional parameter space may be reduced to a two dimensional parameter space. The optimal electrode length is approximately $L_{p} \simeq L / 2$ as the bending moment equals zero at $x=L / 2$ for vertical accelerations. For horizontal accelerations, this point is slightly closer to the frame and some further optimizations regarding the top electrode length is possible.

The objectives for the design are typically a set of soft and hard design criteria to be met, such as matched high sensitivities, a minimum bandwidth say $f_{v}=\omega_{v} / 2 \pi \simeq 25 \mathrm{kHz}$, a suitable capacitance level to ensure immunity to stray capacitance, say $C_{\mathrm{PZT}} \simeq 10$ or $50 \mathrm{pF}$, and a simple fabrication process. The suggested design strategy is as followed.

1) The sensitivity increases with the active PZT volume as long as the strain in the volume has the same sign, thus the optimal PZT film thickness corresponds to a neutral plane at the silicon/PZT interface, thus $H_{p}=\gamma H$.

2) The sensitivity increases as the mass of the seismic mass is increased, thus a thick seismic mass is preferable, however, available silicon wafers are limited in thickness, thus $H_{m}=1000 \mu \mathrm{m}$.

3) The device capacitance is proportional to $W L_{p} / H_{p} \propto$ $W L / H$; a fixed capacitance level, therefore, reduces the number of free parameters such that $W \propto H / L$.

4) The angular resonant frequency of the vertical mode is dependent on five geometrical dimensions; by use of the capacitance, we have $\omega_{v}^{2} / C_{\mathrm{PZT}} \propto H^{4} /\left(L^{4} W_{m} H_{m}\right)$, where $H_{m}$ is already fixed. The set of free design parameters is now reduced to beam thickness $H$ and length $L$.

5) The design is completed by searching numerically for $\Gamma=1$ in equation (35) using $H$ and $L$ as free variables, maybe with practical restrictions such as a desirable PZT thickness.

Table IV shows parameters for two devices designed using this procedure. For these devices the capacitances are $10 \mathrm{pF}$ and $50 \mathrm{pF}$, respectively. The thickness of the silicon beam is taken as $10 \mu \mathrm{m}$ since this would give a optimal PZT thickness of $\sim 14.2 \mu \mathrm{m}$ which is about the minimum thickness possible with the screen printing technique provided by InSensor A/S. In Table IV, excellent agreement between the performance calculated using the analytical and FEM model is seen.
TABLE IV

Dimensions of Two DiFFERENT TRIAXIAL ACCELEROMETERS With the SAME Resonance FREQUenCy BUt DifFERENT Charge Sensitivity and Voltage Sensitivity

\begin{tabular}{l|c|c|c|c|c}
\hline \hline & $\# 1_{\text {Ana }}$ & $\# 1_{\text {FEM }}$ & $\# 2_{\text {Ana }}$ & $\# 2_{\text {FEM }}$ & \\
\hline \hline$C_{0}$ & 10 & 10 & 50 & 50 & {$[\mathrm{pF}]$} \\
\hline$f_{\mathrm{v}}$ & 25 & 23.65 & 25 & 24.58 & {$[\mathrm{kHz}]$} \\
\hline$f_{\mathrm{h}}$ & - & 22.2 & - & 22.7 & {$[\mathrm{kHz}]$} \\
\hline \hline$H$ & 10 & 10 & 10 & 10 & {$[\mu \mathrm{m}]$} \\
\hline$H_{p}$ & 14.2 & 14.2 & 14.2 & 14.2 & {$[\mu \mathrm{m}]$} \\
\hline$H_{m}$ & 1000 & 1000 & 1000 & 1000 & {$[\mu \mathrm{m}]$} \\
\hline$W_{m}$ & 1872 & 1872 & 1413 & 1413 & {$[\mu \mathrm{m}]$} \\
\hline$W$ & 261 & 261 & 758 & 758 & {$[\mu \mathrm{m}]$} \\
\hline$L$ & 193 & 193 & 331 & 331 & {$[\mu \mathrm{m}]$} \\
\hline$\overline{S_{\mathrm{V}_{\mathrm{v}}}}$ & 2.75 & 2.85 & 0.927 & 0.950 & {$[\mathrm{mV} / \mathrm{g}]$} \\
\hline$S_{\mathrm{V}_{\mathrm{h}}}$ & 2.75 & 2.87 & 0.927 & 0.930 & {$[\mathrm{mV} / \mathrm{g}]$} \\
\hline$S_{\mathrm{Q}_{\mathrm{v}}}$ & 0.0275 & 0.0285 & 0.0463 & 0.0475 & {$[\mathrm{mC} / \mathrm{g}]$} \\
\hline$S_{\mathrm{Q}_{\mathrm{h}}}$ & 0.0275 & 0.0287 & 0.0463 & 0.0465 & {$[\mathrm{mC} / \mathrm{g}]$} \\
\hline $\bar{\Gamma}$ & 1 & 1.01 & 1 & 0.98 & \\
\hline
\end{tabular}

\section{CONClusion}

An analytical model of a triaxial PZT MEMS accelerometer based on a single seismic mass suspended by four beams is presented. The analytical model includes the mechanical effect of the piezoelectric layer which is essential for modeling accelerometers based on thick films. For horizontal accelerations the torsion in the two beams orthogonal to the direction of the acceleration is included. For the two beams along the direction of the acceleration both the bending of the beam and the compression and the elongation, respectively, of the beams are included in the model. The model is verified by comparison to a FEM model and excellent agreement between the models is demonstrated, proving that the assumptions used in the analytical model are valid.

\section{APPENDIX}

All the coefficient matrixes used in the analytical and FEM models are listed in this appendix [16]

$$
\begin{aligned}
{[S]_{S i}=} & {\left[\begin{array}{llllll}
7.6 & -2.1 & -2.1 & 0 & 0 & 0 \\
-2.1 & 7.6 & -2.1 & 0 & 0 & 0 \\
-2.1 & -2.1 & 7.6 & 0 & 0 & 0 \\
0 & 0 & 0 & 12.5 & 0 & 0 \\
0 & 0 & 0 & 0 & 12.5 & 0 \\
0 & 0 & 0 & 0 & 0 & 12.5
\end{array}\right] } \\
& \times 10^{-12} \frac{\mathrm{m}^{2}}{\mathrm{~N}} \\
{[S]_{\mathrm{PZT}}=} & {\left[\begin{array}{llllll}
16 & -5.3 & -7.2 & 0 & 0 & 0 \\
-5.3 & 16 & -7.2 & 0 & 0 & 0 \\
-7.2 & -7.2 & 23 & 0 & 0 & 0 \\
0 & 0 & 0 & 60 & 0 & 0 \\
0 & 0 & 0 & 0 & 60 & 0 \\
0 & 0 & 0 & 0 & 0 & 42.6
\end{array}\right] \times 10^{-12} \frac{\mathrm{m}^{2}}{\mathrm{~N}} } \\
{[d]=} & {\left[\begin{array}{llllll}
0 & 0 & 0 & 0 & 300 & 0 \\
0 & 0 & 0 & 300 & 0 & 0 \\
-51 & -51 & 168 & 0 & 0 & 0
\end{array}\right] \times 10^{-12} \frac{\mathrm{C}}{\mathrm{N}} } \\
{[g]=} & {\left[\begin{array}{llllll}
0 & 0 & 0 & 0 & 53 & 0 \\
0 & 0 & 0 & 53 & 0 & 0 \\
-9 & -9 & 39 & 0 & 0 & 0
\end{array}\right] \times 10^{-3} \frac{\mathrm{Vm}}{\mathrm{N}} }
\end{aligned}
$$




\section{REFERENCES}

[1] S. Trolier-Mckinstry and P. Muralt, "Thin film piezoelectrics for MEMS," J. Electroceramics, vol. 12, no. 1-2, pp. 7-17, 2004.

[2] P. Scheeper, J. O. Gulløv, and L. M. Kofoed, "A piezoelectric triaxial accelerometer," J. Micromech. Microeng., vol. 6, pp. 131-133, 1996.

[3] G. H. Gautschi, Piezoelectric Sensorics. New York: Springer, 2002.

[4] J. C. Robinson, J. M. Rybak, and E. R. Saller, "Using accelerometers to monitor complex machinery vibration," Sensors, pp. 36-42, Jun. 1997.

[5] K. Kunz, P. Enoksson, and G. Stemme, "Highly sensitive triaxial silicon accelerometer with integrated PZT thin film detectors," Sens. Actuators, vol. A92, pp. 156-160, 2001.

[6] M. Zhu, P. Kirby, and M. Lim, "Modelling of a tri-axial micro-accelerometer with piezoelectric thin film sensing," in Proc. IEEE Sensors, 2003, pp. 1239-1244.

[7] R. P. van Kampen and R. F. Wolffenbuttel, "Modeling the mechanical behaviour of bulk micro-machined silicon accelerometer," Sens. Actuators, vol. A64, pp. 137-150, 1998.

[8] J. Yu and C. Lan, "System modelling of microaccelerometer using piezoelectric thin films," Sens. Actuators, vol. A 88, pp. 178-186, 2001.

[9] S. P. Beeby, J. N. Ross, and N. M. White, "Design and fabrication of a micromechanical silicon accelerometer with thick-film printed PZT," J. Micromech. Mircoeng., vol. 10, pp. 322-328, 2000.

[10] C. C. Hindrichsen, E. V. Thomsen, R. Lou-Møller, and T. Bove, "MEMS accelerometer with screen printed piezoelectric thick film," in Proc. 5th IEEE Conf. Sensors, 2007, pp. 1477-1480.

[11] R. Lou-Moeller, C. C. Hindrichsen, L. H. Thamdrup, T. Bove, E. Ringgaard, A. F. Pedersen, and E. V. Thomsen, "Screen-printed piezoceramic thick films for miniaturised devices," J Electroceramics, vol. 19, no. 4, pp. 333-338, 2007.

[12] T. Pedersen, C. C. Hindrichsen, R. Lou-Møller, K. Hansen, and E. V. Thomsen, "Investigation of top/bottom electrode and diffusion barrier layer for PZT thick film MEMS sensors," in Proc. IEEE Sensors, 2007, pp. 756-759.

[13] B. Jaffe, W. R. Cook, and H. Jaffe, Piezoelectric Ceramics. New York: Academic Press, 1971.

[14] S. P. Timoshenko and J. N. Goodier, Theory of Eleasticity. New York: McGraw-Hill, 1970

[15] W. G. Young, ROARK'S Formulas for Stress and Strain, 6th ed. New York: McGraw-Hill, 1989.

[16] Properties of Silicon, INSPEC. New York, The Institution of Electrical Engineers, 1988.
Christian Carstensen Hindrichsen received the B.A. degree and the M.Sc. degree in applied physics from the Technical University of Denmark (DTU), Lyngby, Denmark, in 2004 and 2006, respectively. He is currently working towards the Ph.D. degree in the field of PZT-based accelerometers under the supervision of E. V. Thomsen at the Department of Micro- and Nanotechnology (DTU Nanotech).

Ninia S. Almind received the B.A. degree in nanotechnology from the Technical University of Denmark (DTU), Lyngby, Denmark, in 2007. In her B.A project, she worked on fabricating and modeling a PZT-based accelerometer.

Simon H. Brodersen received the B.A. degree in nanotechnology from the Technical University of Denmark (DTU), Lyngby, Denmark, in 2007. In his B.A. project, he worked on fabricating and modeling a PZT-based accelerometer.

Ole Hansen received the M.Sc. degree from the Semiconductor Laboratory, Technical University of Denmark (DTU), Lyngby, Denmark, in 1977.

Since 1977, he has done research in silicon-based micro- and nanotechnology and its applications, first at the Semiconductor Laboratory, and later at the Department of Micro and Nanotechnology (DTU Nanotech). Current research interests include deep reactive ion etching of silicon, $\mathrm{Si}_{1-x} \mathrm{Ge}_{x} / \mathrm{Si}$ heterostructures, microreactors for catalysis, and cantilever-based microprobes for nanoscience. He is presently teaching three lecture courses: Semiconductor Technology, Semiconductor Devices and Microelectromechanical Systems. Since 1990, he has been an Associate Professor at DTU Nanotech.

Erik Villain Thomsen received the M.Sc. degree in physics from Odense University, Odense, Denmark, and the Ph.D. degree in electrical engineering from the Technical University of Denmark (DTU), Lyngby, Denmark.

$\mathrm{He}$ is group leader for the MEMS Applied Sensors activities at the MEMS section, Department of Micro- and Nanotechnology (DTU Nanotech), DTU, where he is affiliated as an Associate Professor. He has been affiliated with DTU Nanotech since 1992 and has been working with fabrication of semi-conductor devices. His current interests include research and teaching within micromechanical multisensors, piezo resistivity of strained layers, piezoelectric MEMS devices, and microsystem packaging. He teaches classes in solid-state electronics, microtechnology and nano- and microfabrication. 